\title{
Survey of knowledge about the diagnosis and treatment of tuberculosis amongst final year medical students and general practitioners in Libreville (Gabon)
}

\author{
Ulrich Davy Kombila ${ }^{1,4 *}$, Dieudonné Mounguengui ${ }^{2,4}$, Irène Mistoul ${ }^{3}$ and Jean Bruno Boguikouma ${ }^{1,4}$ \\ ${ }^{1}$ Internal medicine unit, university teaching hospital center of Libreville, Gabon \\ ${ }^{2}$ Polyvalent medicine unit, military hospital of Libreville, Gabon \\ ${ }^{3}$ Infectious disease unit, university teaching hospital center of Libreville, Gabon \\ ${ }^{4}$ Department of internal medicine, University of medical science, Libreville, Gabon
}

\begin{abstract}
Introduction: Equipping physicians with the competence to manage tuberculosis in Gabon is not just imperative but also urgent as country have been consistently listed as one of the African region where the incidence of disease is very high. However, there were no baseline studies done on knowledge of final year medical students and general practitioners on various aspects of TB diagnosis and management.

Material and method: cross-sectional study by self-administered questionnaire on tuberculosis was filled out by 104 physicians (81 final years' medical students and 23 general practitioners) over a period of activity from 1st to 28th February 2018.

Results: Bacteriological diagnosis of pulmonary tuberculosis was the preferred method of diagnosis among $73.1 \%$ ( $\mathrm{n}=76$ ) physicians. The combination of four tuberculous drugs was selected by $95.2 \%$ of them. For treatment of newly diagnosed tuberculosis, $91.3 \%$ prescribed RHEZ. Preferred treatment durations were 6 $(87.5 \%)$ and $9(10.6 \%)$ months. For patients with treatment failure, $6.7 \%$ and $2.9 \%$ of the physicians would add respectively one and two news drugs to the existing regimen, while $74.0 \%$ would refer the patient to a specialized tuberculosis unit. For tuberculosis during pregnancy $11.5 \%$ of physicians offered treatment according to the national program of fight again tuberculosis, while $20.2 \%$ offered treatment after pregnancy. For treatment of childhood tuberculosis, $40.4 \%$ of physicians thought a three-drug treatment was adequate. For multidrug-resistant tuberculosis $61.5 \%$ claimed to have already heard about it, while none knew the exact definition. To do rapid confirmation of multidrug-resistant TB, XpertMTB/Rif were preferred method of diagnosis among $16.4 \%$ of physicians. DOT is one of the five points of the World Health Organization's (WHO) DOTS (Directly Observed Treatment Short course) strategy for effective TB control, but none of them knew the exact meaning of the DOT sign.
\end{abstract}

Conclusion: The knowledge of physicians about diagnosis and tuberculosis was deemed insufficient. Additional training of students and general practitioners could contribute to improvements in tuberculosis control.

\section{Introduction}

According to the World Health Organization (WHO), the most cost-effective public health measure for TB control is the identification and cure of infectious cases, i.e. patients with pulmonary TB smear positive [1]. Among the five African countries most affected by tuberculosis, Gabon ranks third with an annual incidence of 428 cases per 100,000 inhabitants behind Zimbabwe (562/100000 inhabitants) [2]. The factors that contribute to increasing the incidence and prevalence of tuberculosis in Gabon are the poor performance of the National Tuberculosis Program (NTP) as a cover for DOTS strategy, detection of new cases of bacilli sputum smear [3]. On the other hand, the under-diagnosis of tuberculosis and the inappropriate treatment translate into ongoing local transmission and emergence of resistance, while endangering global control efforts and global health. [4]. Multidrug resistant of tuberculosis from Gabon were initially described by Mounguengui D et al. [5] and confirmed by Belard S et al. [4]. It is clear that tuberculosis control program to be effective, physicians should acquire sufficient knowledge and appropriate attitudes that are essential for management of tuberculosis [6]. The course of medical studies in Gabon was designed to provide training that makes students operational as soon as they leave. The organization of studies, which reconciles theoretical and practical training, is done in such a way as to complete most of the theoretical courses during the first five years [7]. Thus, the last two years must be used to give the student a perfect mastery of clinical situations he will face in medical health training, and to consolidate his theoretical training [7]. It is for this reason that students in final year medical student are interned, referring to hospital interns. They are authorized to provide clinical services to patients presenting at hospitals. They must therefore have the knowledge, skills and attitudes necessary for the proper management of tuberculosis, in line with recommendations of the National Tuberculosis Program (NTP), in a region where tuberculosis remains a real disease public

${ }^{\star}$ Correspondence to: Ulrich Davy Kombila, Internal medicine unit, University teaching hospital center of Libreville, Gabon, Department of internal medicine, Faculty of health science, Libreville, Gabon, PO Box: 9264 Libreville, Tel: (+241) 051806 68, E-mail: ukombila@yahoo.fr

Key words: Tuberculosis, Diagnosis, Treatment, Physicians, Medical student

Received: May 30, 2018; Accepted: June 20, 2018; Published: June 25, 2018 

(Gabon)

health problem. Appropriate knowledge and practice of physicians is crucial for the early diagnosis, treatment and follow-up of TB patients. This is essential for the prevention of resistant TB and the effectiveness of the TB control program. Various factors including experience in managing TB patients, lack of awareness of national TB control guidelines, and the educational background of practitioners affect the standard management of TB patients [8]. In Gabon, we didn't have study has been conducted on the approaches to the diagnosis and treatment of tuberculosis in practitioners. This knowledge assessment is needed to help the NTP design and develop effective control and surveillance strategies to improve screening, early diagnosis and management. Given the paucity of available data on the assessment of knowledge in the approaches to the diagnosis and treatment of tuberculosis of practitioners, it is legitimate to ask whether the NTP recommendations on diagnosis and treatment tuberculosis developed by experts are well known. The purpose of this study was to provide an overview of the approaches to the diagnosis and treatment of tuberculosis of the final year medical student of the Libreville University of Health sciences and general practitioners in Libreville, Gabon.

\section{Patients and Method}

A cross-sectional study was conducted in over a period of activity from $1^{\text {st }}$ to 28 February 2018 at university teaching hospital of Libreville (CHUL). There were 81 final years' medical students and 23 general practitioners. All of them were considered as a physician is this study.

The physicians were evaluated on tuberculosis by self-administered questionnaire with an interviewer. Questionnaire included limited demographic information and questions about knowledge and items about attitudes and practices. Knowledge was assessed with questions on general knowledge, transmission, diagnosis, treatment of TB and knowledge about the latest rapid diagnostic of MDR-TB. Attitude and practice items were based on Gabonese guidelines for control of TB. The questionnaire was administered to all final-year medical students during their clinical rotation and general physicians at university teaching hospital of Libreville. Physicians were asked to answer without referring to any resources for responding to the question. All participants had heard about TB through different sources. Participant was on a voluntary basis and the questionnaire was anonymous.

\section{Statistical analysis}

Data are presented as mean \pm SD. Statistical analysis was performed by $\chi^{2}$ test, using Epi info v 3.5.4 software. P values under 0.05 were considered statistically significant.

\section{Ethical approval}

Respect for the dignity and freedom of final year medical student and general practitioner answering the questions were observed. The confidentiality between the participant and the investigator was also observed by administering an anonymous questionnaire. All study participants provided their informed consent before the start of the study.

\section{Results}

One hundred and four out of 120 final year medical students and general practitioners responded to the distributed questionnaires (86.7\%). The respondent's age ranged between 22 and 37 years with a mean age $28 \pm 2.9$ year, from whom 59 (56.7\%) were female and 45 $(43.3 \%)$ were male. For $95.2 \%(n=99)$ tuberculosis was transmitted by cough. Bacteriology was the preferred means of diagnosis for $73.1 \%$ (n $=76$ ) of the physicians (Table 1). For the treatment of newly diagnosed tuberculosis the majority $(\mathrm{n}=99 ; 95.2 \%)$ of the physicians prescribed a four-drug combination (Table 2). The combination selected most often was isoniazid $(\mathrm{H})$, rifampicin $(\mathrm{R})$, ethambutol $(\mathrm{E})$ and pyrazinamid $(\mathrm{P})$ (91.3\%) (Tables 3 and 4 ). The four-drug tuberculosis as recommended by NTP. The preferred treatment durations were $6(87.5 \%)$ and 9 (10.6\%) months. However, for childhood tuberculosis, $40.4 \%$ ( $\mathrm{n}=$ 42) of physicians thought that a three-drug (HRZ) combination was enough, $17.3 \%(\mathrm{n}=18)$ thought that a two-drug combination was enough, and one physician thought a one-drug regimen was enough, while $22.1 \%(n=23)$ of physicians thought that treatment for childhood tuberculosis was similar to that for adult tuberculosis; $19.2 \%$ gave no reply. In case of TB during pregnancy, $11.5 \%$ of physician would offer standard therapy during pregnancy, $12.5 \%$ would offer threedrug combination and would offer treatment after pregnancy; $55.8 \%$ gave no response to this question. According to directly supervised or directly observed treatment ("DOT"), one of the pivot of DOTS strategy advocated by the WHO to effectively control tuberculosis, $98.1 \%(n=102)$ of physicians didn't know the meaning of the acronym "TDO". In case of failure of first-line regimen TB treatment, 74.0\% (n $=77$ ) of the physicians recommended sending them to a specialized tuberculosis unit, while $6.7 \%(n=7)$ said they would add one drug to the existing regimen (Table 5). More than half of physicians, $61.5 \%$ (n $=64$ ), reported having heard of multidrug-resistant TB. However, none of them knew the exact definition of multidrug-resistant (MDR-TB) and ultra-resistant tuberculosis (XDR-TB). To rapidly confirm MDRTB $27.8 \%(\mathrm{n}=29)$ had found it useful to carry out Lowenstein solid medium culture, while $15.4 \%(\mathrm{n}=16)$ of physicians preferred to require to a diagnostic and rapid sensitivity test of molecular biology, XpertMTB/

Table 1. Physicians' approaches to diagnosis of tuberculosis

\begin{tabular}{|c|c|c|}
\hline \multirow{2}{*}{ Diagnosis } & \multicolumn{2}{|c|}{} \\
\cline { 2 - 3 } & $\mathrm{N}(\%)$ & CI 95\% \\
\hline Clinical & $0(0.0)$ & $16.2-33.4$ \\
\hline Clinical + radiographic & $25(24.0)$ & $63.5-81.3$ \\
\hline Bacteriological & $76(73.1)$ & $0.6-8.2$ \\
\hline Other & $3(2.9)$ & \\
\hline
\end{tabular}

Table 2. Number of drugs prescribed by physicians for newly diagnosed tuberculosis

\begin{tabular}{|c|c|c|}
\hline \multirow{2}{*}{ Number } & $\mathrm{N}(\%)$ & CI 95\% \\
\cline { 2 - 3 } & $1(1.0)$ & $0.02-5.2$ \\
\hline One e & $3(2.9)$ & $0.6-8.2$ \\
\hline Three & $99(95.2)$ & $89.1-98.4$ \\
\hline Four & $1(1.0)$ & $0.02-5.2$ \\
\hline Other & & \\
\hline
\end{tabular}

Table 3. Drug combinations prescribed by physicians for newly diagnosed tuberculosis in adult

\begin{tabular}{|c|c|c|}
\hline \multirow{2}{*}{ Drug combinations } & & \\
\cline { 2 - 3 } & $\mathrm{N}(\%)$ & CI95\% \\
\hline HR & $1(0.9)$ & $0.02-5.2$ \\
\hline HRE & $3(2.9)$ & $0.6-8.2$ \\
\hline HRZE & $95(91.3)$ & $84.2-95.9$ \\
\hline HRZ & $3(2.9)$ & $0.6-8.2$ \\
\hline Other & $2(1.9)$ & $0.2-6.7$ \\
\hline
\end{tabular}

Table 4. duration of treatment prescribed by the physicians

\begin{tabular}{|c|c|c|}
\hline \multirow{2}{*}{ Duration } & N (\%) & CI95\% \\
\cline { 2 - 3 } & $91(87.5)$ & $75.5-93.1$ \\
\hline Six mois & $0(0.0)$ & \\
\hline Huit mois & $11(10.6)$ & $5.4-18.1$ \\
\hline Neuf mois & $2(1.9)$ & $0.2-6.7$ \\
\hline Douze mois & & \\
\hline
\end{tabular}



(Gabon)

Rif (Table 6). Among risk situations of developing MDR-TB, 88.5\% $(\mathrm{n}=92)$ of physicians thought that relapse after treatment were a risk situation, $80.8 \%(\mathrm{n}=84)$ thought failure were a risk situation and $62.5 \%$ $(n=65)$ thought people living with HIV were a risk situation (Table 7).

\section{Discussion}

Tuberculosis is almost always curable if patients are given adequate treatment. Despite this, however, tuberculosis has provided impossible to eliminate, and the number of drug-resistant cases has increased due to physicians' and patents' failure to comply with recommended treatment regimens. The study revealed a lack of in the knowledge of MDR-TB management according to NTP. The most critical points identified included the definitions of MDR-TB, XDR-TB and the acronym "DOT". Many patients receive self-administered treatment on their own responsibility and do not follow it. It is not possible to predict who will follow or not follow his treatment; this is why treatment under Directly Observed Treatment (DOT), outpatient is necessary in order to ensure a good compliance of the TB treatment which represents a key factor for success. In fact, concerning MDR - TB, $61.5 \%$ of physicians were had already heard about it. However, none of them has been able to give an exact definition of MDR - TB and XDR - TB. This finding was also made in a previous study in Turkey by Javed $\mathrm{H}$ et al., where $36.41 \%$ of students knew definition of MDR - TB [9]. MDR - TB, which most often results from poor compliance with TB-drug. It is particularly dangerous form of tuberculosis caused by resistant of Mycobacterium tuberculosis to at least isoniazid and rifampicin, the two most effective major TB drugs [10]. This MDR - TB can lead to the development of extremely resistant strains (XDR - TB), practically untreatable [11]. This is a direct consequence of the poor management of tuberculosis disease [12]. The severity of these cases, the cost of treatment, the complexity of the drugs used, and the severity of their side effects are

Table 5. Physicians' approach to treatment failure

\begin{tabular}{|c|c|c|}
\hline \multirow{2}{*}{ Approaches } & \multicolumn{2}{|c|}{} \\
\cline { 2 - 3 } & $\mathrm{N}(\%)$ & CI95\% \\
\hline Add only one new drug & $7(6.7)$ & $2.7-13.2$ \\
\hline Add two new drugs & $3(2.9)$ & $0.6-8.2$ \\
\hline $\begin{array}{c}\text { Refer to specialized } \\
\text { tuberculosis center }\end{array}$ & $77(74.0)$ & $64.5-82.1$ \\
\hline Other & $17(16.3)$ & $9.3-23.6$ \\
\hline
\end{tabular}

Table 6. Physicians' approach to rapid diagnosis of MDR-TB

\begin{tabular}{|c|c|c|}
\hline \multirow{2}{*}{ Approaches } & \multicolumn{2}{|c|}{} \\
\cline { 2 - 3 } & $\mathrm{N}(\%)$ & CI95\% \\
\hline Radiological and clinical & $3(2.9)$ & $0.6-8.2$ \\
\hline Zielh Neelsen stain & $20(19.2)$ & $12.2-28.1$ \\
\hline Xpert MTB/Rif & $16(15.4)$ & $9.1-23.7$ \\
\hline CT scan & $17(16.3)$ & $9.8-24.8$ \\
\hline Culture (Lowenstein) & $29(27.9)$ & $19.5-37.5$ \\
\hline Other & $19(18.3)$ & $11.3-27.1$ \\
\hline
\end{tabular}

Table 7. Physicians' approach to people with risk to develop MDR-TB

\begin{tabular}{|c|c|c|}
\hline \multirow{2}{*}{ Approaches } & \multicolumn{2}{|c|}{} \\
\cline { 2 - 3 } & $\mathrm{N}(\%)$ & CI95\% \\
\hline Failure & $84(80.8)$ & $71.8-87.8$ \\
\hline Treatment relapse & $92(88.5)$ & $80.7-93.8$ \\
\hline People living with HIV & $65(62.5)$ & $52.4-71.8$ \\
\hline Mellitus & $21(20.2)$ & $12.9-29.1$ \\
\hline Children under 5 years & $19(18.3)$ & $11.3-27.1$ \\
\hline Medical practitioners & $53(50.9)$ & $40.9-60.9$ \\
\hline Final years student medical & $48(46.1)$ & $33.6-56.2$ \\
\hline
\end{tabular}

all factors that encourage these patients to be referred to a specialized tuberculosis unit [13]. In the case of first-line treatment failure, $74.0 \%$ of the physicians thought it would be useful to send the patient to a specialized tuberculosis unit, while $6.7 \%$ recommended adding one drug to the regimen. The addition of a single drug to a failure regimen is incorrect. Aba Y et al., reported that the common errors in treatment were $22 \%$ of the physicians recommended adding one drug to the regimen, while $50 \%$ sent the patient to a specialized tuberculosis unit [14]. In Turkey $65.5 \%$ of physicians decided to refer patients to the specialized tuberculosis unit in case of failure and 20.2\% recommended adding one drug to the regimen [15]. The addition of TB-drug one by one in case of first-line treatment failure is one of the factors favoring the appearance of MDR-TB strain [12]. To rapidly confirm MDR-TB, $16.4 \%$ of physicians preferred a molecular biology test (Xpert MTB/ Rif). To reduce the time between the detection of tuberculosis and the demonstration of drug resistance, WHO recommends the use of the Xpert MTB/Rif test in suspected MDR-TB cases [16]. In practice, molecular detection of rifampicin resistance is used to detect rapidly resistant strains [12]. Resistance of rifampicin is generally associated with isoniazid resistance in many countries, where rifampicin monoresistance is rare [10]. According to the National Tuberculosis Program (NTP), population risk of MDR-TB are: failures, relapses, positive microscopy control at $3 \mathrm{rd}$ month, close contacts of symptomatic MDRTB cases, and people living with HIV (PLHIV), mellitus, children under 5 years, health workers, prisoners [17]. Among the main people at risk of MDR-TB, physicians thought respectively: failure $(88.5 \%)$, relapse $(80.8 \%)$, and people living with HIV (62.5\%), and medical staff (50.9\%) were population risk to develop MDR-TB. In this study $73.1 \%$ of the physicians depended on mycobacteriological analysis of sputum. However, in a previous study conducted respectively in Turkey [15] and Ivory Cost [14] on approaches to the diagnosis and treatment of tuberculosis $75 \%$ and $2 \%$ preferred a bacteriological diagnosis of sputum for the confirmation of tuberculosis. The confirmation of tuberculosis is bacteriological. Classically it is done by the detection of acid-fast bacilli (AFB) on microscopic examination after staining with Ziehl - Neelsen or auramine (fluorescence microscopy) which is a fast, inexpensive, sufficient and specific technique in regions of high incidence [18]. From a public health point of view, adequate treatment of patients, with positive AFB, is the most effective measure to control tuberculosis infection [19]. The world Health Organization (WHO) recommends a 6 or 8 month short-course regimen with four drugs in the initial phase (HRZ and S (streptomycin) or E for two months) and two drugs in the continuation phase (HR for 4 months or HE for 6 months) in news cases (Category 1) [15]. In this study the drug combination most often selected was HRZE (91.3\%). According to the NTP, this prescription was appropriated [20]. The standard regimen consists of four drugs divided into two phases: an intensive phase combining four-drugs (HREZ) for 2 months followed by a consolidation phase associating two drugs for 4 months [19], for an overall duration of six months as proposed by $87.5 \%$ physicians. About childhood TB, $40.4 \%$ of the physicians thought that HRZ was the treatment combination, while $22.1 \%$ of the physicians thought that treatment of childhood tuberculosis was similar to that for adults. In practice, childhood TB is treated with a 6 month regimen consisting of HR, with the addition of $\mathrm{Z}$ in the first months. If primary resistance is high, $\mathrm{E}$ is added to the regimen [15]. But ethambutol (E) was not recommended by the NTP [20] for childhood under 30 weights due to ocular complications related with use of ethambutol. In case of association of $\mathrm{TB}$ - pregnancy, $11.5 \%$ of physicians offered standard treatment during pregnancy, $12.5 \%$ offered treatment with three-drug (HRZ) combination, while $20.2 \%$ offered treatment after the completion of pregnancy. Standard 

(Gabon)

anti-tuberculosis treatment is applied during pregnancy, excluding SM and other aminoglycosides, as this agent are ototoxic for the foetus $[15,20]$. There is an urgent need for massive increase in awareness of DOTS, MDR - TB and XDR - TB among medical students and general practitioners. The revision of existing medical education curriculum in Gabon should focus on incorporation of national TB guidelines into TB teachings in schools. The appropriate authority should ensure the circulation and availability of TB guidelines to every practicing medical doctor in the country. This will encourage medical practitioners to inculcate diagnostic and prescription practices that are in accordance with the national TB guidelines [21].

\section{Conclusion}

This study highlights many shortcomings. This requires the implementation of corrective actions in the field of tuberculosis control through initial and continuing medical training aimed first of all at raising the level of their knowledge of tuberculosis and at the same time improving their knowledge of tuberculosis competence for proper management of the disease according to NTP recommendations.

\section{References}

1. Le traitement de la tuberculose: principes à l' intention des programmes nationaux. WHO_CDC_TB_2003.313_Fr.

2. Mjid M, Cherif J, Ben Salah N, Toujani S, Ouahchi Y, et al. (2015) Tuberculosis epidemiology. Rev Pneumol Clin 71: 67-72. French article.

3. Toung Mve M, Bisvigou U, Diop Barry NC, Ella Ondo C, Nkoghe D (2010) Reasons for stopping and restarting tuberculosis treatment in Libreville (Gabon). Cahiers Santé 20; 31-34.

4. Bélard S, Remppis J, Bootsma S, Janssen S, Kombila DU, et al. (2016) Tuberculosis Treatment Outcome and Drug Resistance in Lambaréné, Gabon: A Prospective Cohort Study. Am J Trop Med Hyg 95: 472-480. [Crossref]

5. Mounguengui D, Ondounda M, Mandji Lawson JM, Fabre M, et al. (2012) Multiresistant tuberculosis at the hôpital d'instructions des armées de Libreville (Gabon) about 16 cases. Bull Soc Pathol Exot 105: 1-4. [Crossref]

6. Behnaz F, Mohammadzade G, Mousavi-e-Roknabadi RS, Mohammadzadeh M (2014) Assessment of knowledge, attitudes and practices regarding tuberculosis among final year students in Yazd, Central Iran. J Epidemiol Glob Health 4: 81-85. [Crossref]
7. Theraplix Guide to Medical Studies (1982) Student Guide of the University Health Sciences Center. Unpublished document. French publication.

8. Yimer SA, Holm-Hansen C, Bjune G (2012) Assessment of knowledge and practice of private practitioners regarding tuberculosis control in Ethiopia. J Infect Dev Ctries 6: 13-19. [Crossref]

9. Javed H, Tahir Z, Hashmi HJ, Jamil N (2016) A cross-sectional study about knowledge and attitudes toward multidrug-resistant and extensively drug-resistant tuberculosis in a high-burden drug-resistant country. Int J Mycobact 5: 128-134. [Crossref]

10. Tritar F, Daghfous H, Ben Saad S, Slim-Saidi L (2015) [Management of multidrugresistant tuberculosis]. Rev Pneumol Clin 71: 130-139. [Crossref]

11. Zellweger JP (2011) Multidrug resistant tuberculosis - its extent, hazar and possible solution. Rev Mal Respir 28: 1025-1033. [Crossref]

12. Vérizis N, Robert J (2003) Tuberculose multi-résistante : prise en charge. La Lettre de l'Infectiologue - Tome XVIII 5: 186-191.

13. Antoun F, Vérizis N (2011) Managing multidrug-resistant tuberculosis. Rev Mal Respir 28: 956-957.

14. Aba Y, Domoua K, Daix T, Bakayoko A, Yapi A, et al. (2007) Approaches to Tuberculosis Diagnosis and Treatment of Seventh-Year Students in the Abidjan Medical Education and Research Unit (Ivory Coast). Rev Pneumol Trop 7: 30-33.

15. Cirit M, Orman A, Unlu M (2003) Physicians' approach to the diagnosis and treatment of tuberculosis in Afyon, Turkey. Int J Tuberc Lung Dis 7: 243-247. [Crossref]

16. World Health Organization (2012) Tuberculosis diagnostics XpertMTB/Rif Test. WHO endorsement and recommendations. WHO.

17. National Tuberculosis Program (2016) National Technical Guide for the Management of Drug-Resistant Tuberculosis in Gabon. 1ère Edition, December. Unpublished document.

18. Toujani S, Ben Salah N, Cherif J, Mjid M, Ouahchy Y, et al (2015) Primary and pulmonary tuberculosis. Rev Pneumol Clin 71: 73-82.

19. Ben Amar J, Dhahri B, Aouina H, Azzabi S, Baccar MA, et al. (2015) [Treatment of tuberculosis]. Rev Pneumol Clin 71: 122-129. [Crossref]

20. National Tuberculosis Program (2010) Training Modules for Health Providers in Tuberculosis Case Management: Participant Manual. Edition, November. Unpublished documen. French publication

21. Olakunle OS, Oladimeji O, Olalekan AW, Olugbenga-Bello A, Akinleye C, et al. (2014) Knowledge of tuberculosis management using directly observed treatment short course therapy among final year medical students in South Western Nigeria. Pan Afr Med J 18:32 [Crossref]

Copyright: (C2018 Kombila UD. This is an open-access article distributed under the terms of the Creative Commons Attribution License, which permits unrestricted use, distribution, and reproduction in any medium, provided the original author and source are credited. 